\title{
The expression and distribution of aquaporin 3 in mouse embryos before and after vitrification
}

\author{
Ying-qi Nong • Feng-hua Liu $・$ Ye Chen $\cdot$ Fang Wang
}

Received: 29 November 2012 / Accepted: 25 February 2013 /Published online: 17 March 2013

(C) The Author(s) 2013. This article is published with open access at Springerlink.com

\begin{abstract}
Purpose To determine the role of aquaporin 3 (AQP3) isoform in early embryonic development and protecting embryos subjected to freeze-thawing for assisted reproduction, we investigated the expression and distribution of AQP3 in mouse embryos at different developmental stages before and after vitrification.

Methods Eight-cell embryos, morulae, and blastocysts were obtained from female mice that had been superovulated by controlled ovarian hyperstimulation. Immunofluorescence staining, laser confocal microscopy, and Western blot were used to determine the expression and distribution of AQP3 in preimplantation mouse embryos before and after vitrification.

Results AQP3 was expressed at the 8-cell to blastocyst stage before and after vitrification. The expression and distribution of AQP3 was developmentally regulated at the 8-cell to blastocyst stage. The expression of AQP3 was significantly decreased in 8-cell embryos and early blastocysts after vitrification. However, at the morulae stage, the expression of AQP3 was increased after vitrification. Conclusions The developmental and vitrification-dependent changes in AQP3 expression and distribution suggest that this transmembrane channel might regulate mouse embryo development and contribute to the protective response during vitrification.
\end{abstract}

Keywords Aquaporin 3 - Mouse embryo · Vitrification · Immunofluorescence

Capsule AQP3 might regulate mouse embryo development and contribute to the protective response during embryo vitrification.

Y.-q. Nong $\cdot$ F.-h. Liu $(\bowtie) \cdot$ Y. Chen $\cdot$ F. Wang

Department of Reproductive Medicine, Guangdong Women and Children Hospital, No. 13 Guangyuan West Road, Yuexiu District, 510010, Guangzhou, Guangdong, China

e-mail: liushine2006@163.com

\section{Introduction}

Assisted reproductive technologies (ARTs) typically require frozen-thawed embryo transfer (F-ET) to facilitate pregnancy during controlled ovarian hyperstimulation $(\mathrm{COH})$ cycles. The transmembrane flux of water and cryoprotectant is a key factor affecting the viability of embryos following freeze-thawing, and AQPs are the main molecular pathways through which water and cryoprotectants permeate embryonic cells [1].

Aquaporins (AQPs) channels are widely distributed in animals and plants where they mediate the transmembrane movement of water to maintain cellular fluid and osmotic balance. So far, at least 12 homologous members (AQPO AQP12) have been molecularly identified in mammals [2], they are now subdivided into two major groups [3]: orthodox AQPs and aquaglyceroporins. Aquaglyceroporins includes five members: AQP3, AQP7, AQP8, AQP9 and AQP10, they mediate the transmembrane movement of ethylene glycol, glycerol, ureophil, hydroxyl compounds, and other small molecules in addition to water [4]. A previous study [5] reported that AQPs are highly expressed in the mammalian reproductive system and actively participate in multiple reproductive processes, including follicle growth, spermatogenesis, embryo formation, embryo development, and implantation.

Previous studies [6,7] suggested that AQPs especially aquaglyceroporins may provide a protective effect during embryonic freeze-thawing. AQP3 channel is a member of aquaglyceroporins and several independent investigations [8-10] demonstrated that AQP3 is highly expressed in mouse embryos of all early developmental stages. To determine the role of this AQP isoform in early embryonic development and protecting embryos subjected to freezethawing for assisted reproduction, we investigated the 
expression and distribution of AQP3 in preimplantation mouse embryos before and after vitrification.

\section{Materials and methods}

Mouse embryo collection

Embryos were obtatined from female KM mice (age 5-8 wk, weight 25-30 g; Medical Experimental Animal Center of Guangdong Province) that had been superovulated by intraperitioneal injection of $5 \mathrm{IU}$ pregnant mare serum gonadotropin (PMSG, DHY Pharm, China) followed $47 \mathrm{~h}$ by $5 \mathrm{IU}$ chorionic gonadotropin (hCG, Livzon, China). Immediatedly after hCG, the females were caged overnight with KM males (Medical Experimental Animal Center of Guangdong Province). Successful mating was determined the following morning (day 1) by detection of a vaginal plug. The time of post-hCG was used to measure the embryo developmental stage.

Preimplantation mouse embryos were collected at $65-68 \mathrm{~h}$ (the 8-cell stage), 80-85 h (the morulae stage), and $90 \mathrm{~h}$ (the blastocyst stage) post-hCG. Embryos were flushed from the reproductive tract and uterus employing phosphate buffer (PBS) containing $0.05 \%$ fetal bovine serum (FBS) by the literature method [11]. Embryos were washed four to five times in flushing medium, transferred to $10 \mu \mathrm{l}$ microdrop of G-1 (Vitrolife) culture medium under paraffin oil (Amresco), and cultured under $5 \% \mathrm{CO}_{2}$ in air atmosphere at $37^{\circ} \mathrm{C}$.

\section{Embryo vitrification freezing}

At room temperature, embryos were transfered to multiblast medium (BM) containing PBS with $20 \%$ FBS for a few seconds. And then, embryos were suspended first in vitrification solution 1 (VS1; BM solution containing $7.5 \%$ $\mathrm{v} / \mathrm{v}$ DMSO and $7.5 \% \mathrm{v} / \mathrm{v}$ ethylene glycol) for $2 \mathrm{~min}$, then in 3-4 drops of VS2 (BM solution containing $15 \% \mathrm{v} / \mathrm{v}$ DMSO, $15 \% \mathrm{v} / \mathrm{v}$ ethylene glycol, and $10 \% \mathrm{v} / \mathrm{v}$ sucrose) in turns, 40 seconds altogether; and placed to rod, put into liquid nitrogen immediately.

All solutions were warmed in $37{ }^{\circ} \mathrm{C}$ at least $30 \mathrm{~min}$ before application.

\section{Embryo vitrification thawing}

Carried rod with the embryos to be thawed was inserted to warming solution 1 (WS1; BM solution containing $35 \% \mathrm{v} / \mathrm{v}$ sucrose) for $2 \mathrm{~min}$, then the embryos were transfered to WS2 (BM solution containg $25 \% \mathrm{v} / \mathrm{v}$ sucrose) for $3 \mathrm{~min}$, $\mathrm{BM}$ solutions for $5 \mathrm{~min}$, and then $\mathrm{BM}$ solutions again for 5 min, in turns. And then embryos were transferred to G-1 culture medium under paraffin oil, placed to $5 \% \mathrm{CO}_{2}$ incubator at $37{ }^{\circ} \mathrm{C}$ for at least $2 \mathrm{~h}$. All solutions were warmed in $37^{\circ} \mathrm{C}$ at least 30 min before application.

Immunofluorescence for detection of AQP3

Mouse embryos were collected as described above. All procedures were performed at room temperature unless otherwise noted. Briefly, embryo pools were fixed in $4 \%$ paraformaldehyde in PBS for $30 \mathrm{~min}$. Fixed embryos were washed three times in PBS then used immediately, or stored at $4{ }^{\circ} \mathrm{C}$ in Embryo Storage Buffer (PBS $+0.9 \%$ sodium azide) up to 1 week until the next step. Fixed embryos were permeabilized in $0.01 \%$ Triton X-100 and $0.01 \% \mathrm{G}-1$ culture medium in PBS for $30 \mathrm{~min}$, followed by washing three times in fresh PBS, and blocked in the goat serm confining liquid for $1 \mathrm{~h}$. Embryos were incubated with primary antisera for AQP3 (Santa Cruz, America) at a 1:250 dilution in Antibody Dilution/Wash Buffer (ADB; Boster, China) overnight at $4{ }^{\circ} \mathrm{C}$. Embryos were then washed three times for $20 \mathrm{~min}$ in ADB and incubated with fluoresceine isothiocyanate (FITC)-conjugated secondary antibody (1:64; Boster, China) for $1 \mathrm{~h}$, followed by karyotin for $20 \mathrm{~min}$ in $10 \mu \mathrm{l}$ 4,6-diamino-2-phenyl indole (DAPI), then washed twice in ADB. Fully processed embryos were mounted and sealed onto glass slides in $20 \mu \mathrm{l}$ of anti-fade mounting Reagent (Boster, China), $22 \times 22 \mathrm{~mm}$ glass coverslips were slightly put under glass slides. Immunofluorescence images was obtained using a fluorescence microscope (Leica, DM1R13) or a laser-scanning confocal microscopy (ZEISS, LSM710) in conjunction with the Leica Image Capture System. Slides were stored in a light tight box at $-20{ }^{\circ} \mathrm{C}$ up to 2 days. The experiment was repeated three times, every time, 20-30 embryos of each developmental stage were examined.

\section{Western blot analysis}

A total of 150-180 embryos at each stage were collected in loading buffer and frozen at $-80{ }^{\circ} \mathrm{C}$ until use. Whole-cell extracts were prepared using mammalian protein extraction reagent (Beyotime, China) and the protein concentration was determined by BCA assay (Beyotime, China). Samples were separated onto $12 \%$ SDS-polyacrylamide gels. After electrophoresis, proteins were transferred onto a PVDF membrane for $1 \mathrm{~h}$, which were then incubated overnight at $4{ }^{\circ} \mathrm{C}$ in blocking solution containing $5 \%$ non-fat dry milk in PBS with $0.1 \%$ Tween-20 (PBST). Subsequently, the membranes were incubated with the primary antibody in blocking solution overnight at $4{ }^{\circ} \mathrm{C}$, following by an incubated with horseradish peroxidase-conjugated IgG secondary antibodies (1:1000, Boster, China) for $1 \mathrm{~h}$. Membranes were washed three times in PBST and then processed using the enhanced chemiluminescence (ECL) 
detection system. SDS sample buffer was used as negative control. The relative abundance of AQP3 protein was normalized against GAPDH protein. All experiments were repeated at least three times.

\section{Data analysis}

Fluorescence staining and confocal images were analyzed using Zeiss LSM Image Browser Version 4.2.0.121. Within a given experiment, all images used for figures were subjected to identical brightness and contrast adjustmens to optimize visualization, and in no case did this change the visibility of cellular structures. The Image-Pro plus software (6.0 version) was used to quantify the IOD (Integral Optical Density) of AQP3 for Western blot. All data were presented as mean $\pm \mathrm{SE}$, statistical analyses were performed using $T$-test, using SPSS16.0 statistical software for statistical analysis, and their values were considered statistically significant at $P<0.05$.

\section{Statement}

Any human subjects featured in this study have been confirmed with the Helsinki declaration and have followed the guidelines in this investigation. This study was approved by the Cancer Center of Guangzhou Medical University IRB and appropriate institutional animal care and use committee (IACUC).

\section{Result}

Localization of AQP3 on embryos before and after vitrification

Immunoflurescence staining revealed that AQP3 was highly expressed in 8-cell embryos, morulae, and early blastocysts before and after freeze-thaw treatment (Fig. 1).

Laser confocal microscopy revealed distinct spatial expression patterns of AQP3 at different embryonic stages. At the 8-cell stage, the AQP3-specific fluorescence signal was evenly distributed over the cytoplasm and karyotheca of blastomeres, with stronger expression in the karyotheca relative to the cytoplasm (Fig. 2a); In the morula stage, AQP3 was evenly distributed over the plasma membrane of each blastomere (Fig. 2b); In early blastocysts, AQP3 was predominantly distributed over the plasma membrane and cytoplasm of trophoblastic cell, as well as blastocyst cavity (Fig. 2c). After freeze-thawing, at the morula stage, the AQP3 localization
Fig. 1 Expression of AQP3 in mouse early embryo before and after vitrification by immunoflurescence microscopy. Embryos were labeled with DAPI (blue) as shown, as is AQP3 immunolocation (green). In control group, embryos were collected at the 8-cell stage (a), morula stage (b), and blastocyst (c). After vitrification, 8-cell embryo (d), morula (e), and blastocyst (f) were labeled. Examples of negative embryos didn't incubator with primary antibody show absence of AQP3 staining, $(\mathrm{G}, \mathrm{H})(20 \times)$
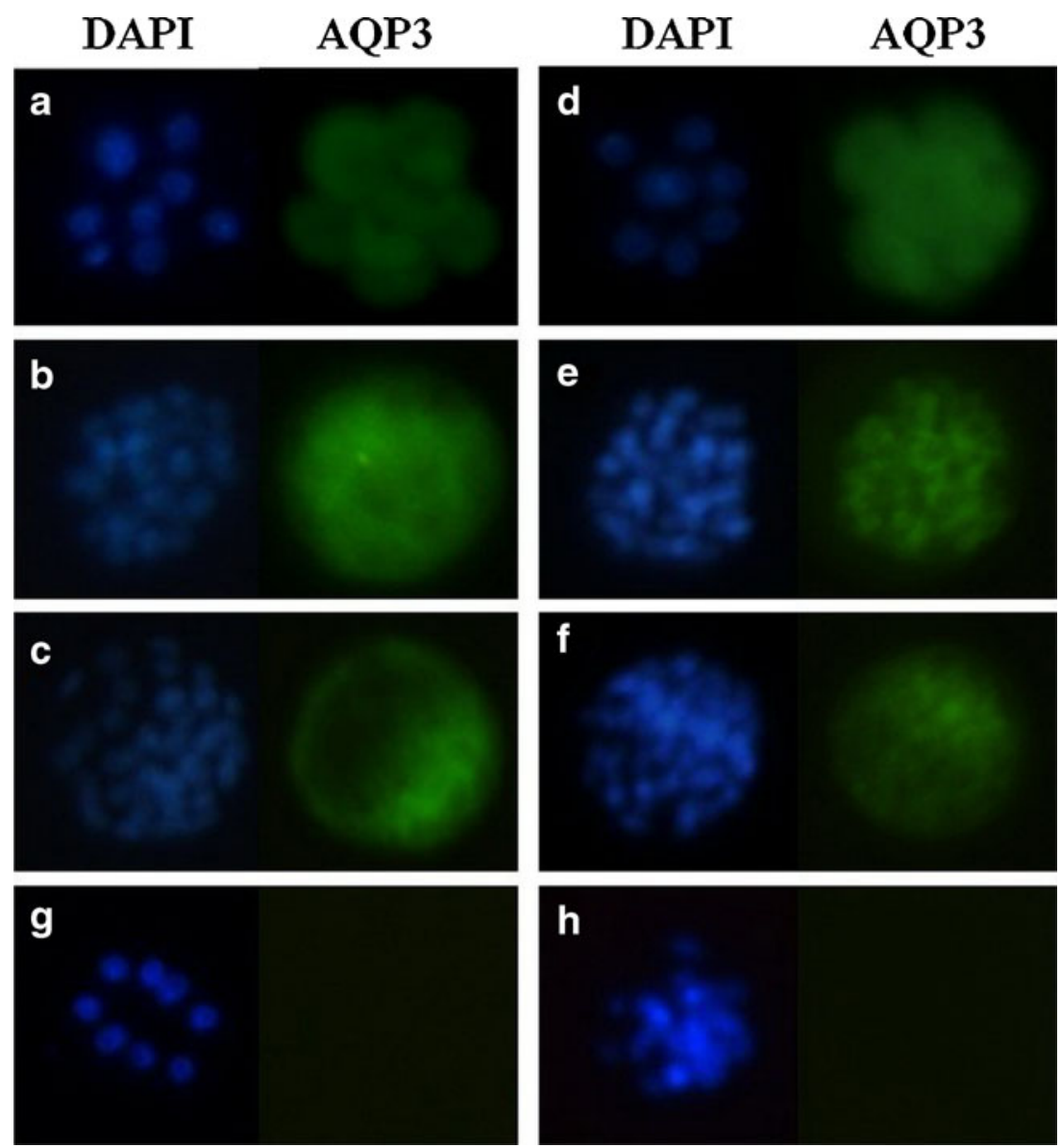

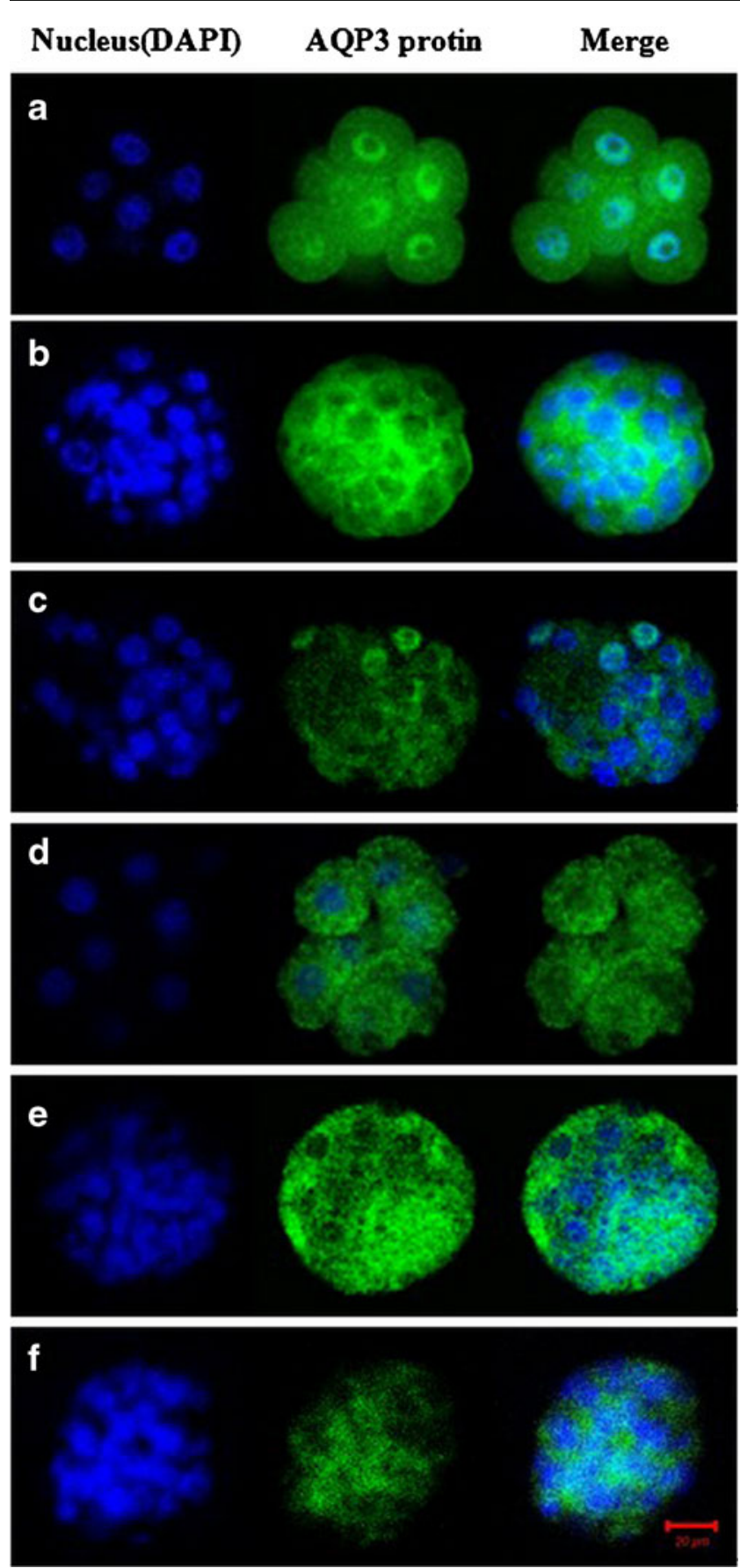

Fig. 2 Localization of AQP3 in mouse early embryos before and after vitrification by laser confocal microscopy. Embryos were labeled with DAPI for DNA (blue; left) and AQP3 immunolocation (green; middle) and merged images (right), as indicated (top). The images shown here are representative images from about 50 independent replicates of embryos treated without vitrification at 8-cell stage (a), morulae stage (b), and blastocyst stage (e), and with vitrification at 8-cell stage (d), morulae stage (e), and blastocyst stage (f). Scale bar $=20 \mu \mathrm{m}$

was the same as the control embryos at morula and blastocyst stage (Fig. 2e, f). However, after vitrification, AQP3 mainly distributed in the cytoplasm, and AQP3 immunofluorescence wasn't detected in karyotheca at 8-cell stage. (Fig. 2d).
The expression levels of AQP3 on embryos before and after vitrification

We measured the expression levels of AQP3 at different embryonic stage before and after vitrification by Western blot analysis. According to statistics, the expression levels of AQP3 before vitrification in 8-cell embryos was higher than after vitrification (IOD $1.394 \pm 0.068$ to $0.573 \pm 0.019$, $P<0.05$ ), as well as blastocysts stage (IOD $1.000 \pm 0.040$ to $0.573 \pm 0.019, P<0.05$ ), while the expression of AQP3 before vitrification in morulae was lower than after vitrification(IOD $0.928 \pm 0.046$ to $1.173 \pm 0.068, P<$ 0.05) (Fig. 3).

\section{Discussion}

Edashige et al. [8] measured AQP1-9 mRNAs in oocytes and early embryos from ICR mice at various development stages and noted that AQP3 and AQP7 mRNAs were highly expressed in MII oocytes, four-cell embryos, morulae, and blastocysts, while AQP8 and AQP9 mRNAs were only expressed in blastocysts. In contrast, AQP1, 2, 4, 5, and 6 were not detected in embryos. Though several subsequent studies $(9,10)$ yielded some discrepant results, all found that AQP3 and AQP7 mRNAs were highly expressed in preimplantation mouse embryos at different stagesand that AQP8 mRNA was highly expressed in early blastocysts, suggesting that AQP3, AQP7, and AQP8 have specific functions in the early stages of embryogenesis. Edashige et al. [12] also examined the distribution of AQP3 and AQP7 and found that AQP3 was expressed at the morula surface but not at the oocyte surface, while both stages expressed little AQP7. In CD1 mice, Barcroft et al. [13] found that the AQP3 protein was expressed in the plasma membranes of 1-8-cell embryos, the outer cells of morulae, the inner cell mass of blastocysts, and at the margins of trophectoderm base cells.

The present study compared the level of AQP3 protein expression in mouse embryos at three developmental stages before and after vitrifcation. Immunoflurescence revealed that AQP3 was highly expressed in 8-cell embryos, morulae, and early blastocysts before and after freezethaw treatment. Laser confocal microscopy revealed distinct spatial expression patterns at these stages. In 8-cell embryos, the AQP3-specific fluorescence signal was evenly distributed over the cytoplasm and karyotheca of blastomeres before vitrification, with stronger expression in the karyotheca relative to the cytoplasm. After freeze-thawing, AQP3 mainly distributed to the cytoplasm, while much less AQP3 immunofluorescence was detected in karyotheca. In the morula stage, AQP3 was evenly distributed over the plasma membrane of each 
Fig. 3 Expression levels of AQP3 as detected by Western blot analysis. Protein levels of AQP3 were normalized against GAPDH. Expression level of AQP3 were detected on before and after embryo vitrification at 8-cell stage(8), morulae stage $(\mathrm{m})$ and blastocyst stage(b), with the characteristic $34 \mathrm{Kda}$ band. The form of 36 $\mathrm{Kda}$ was identified as GAPDH. The calculation results shown the expression level of AQP3 after vitrification(blue bar) was lower before vitrification(green bar) in 8-cell embryos and early blastocysts, whereas that after vitrification was higher than before vitrification in morulae. $(*: P<0.05)$

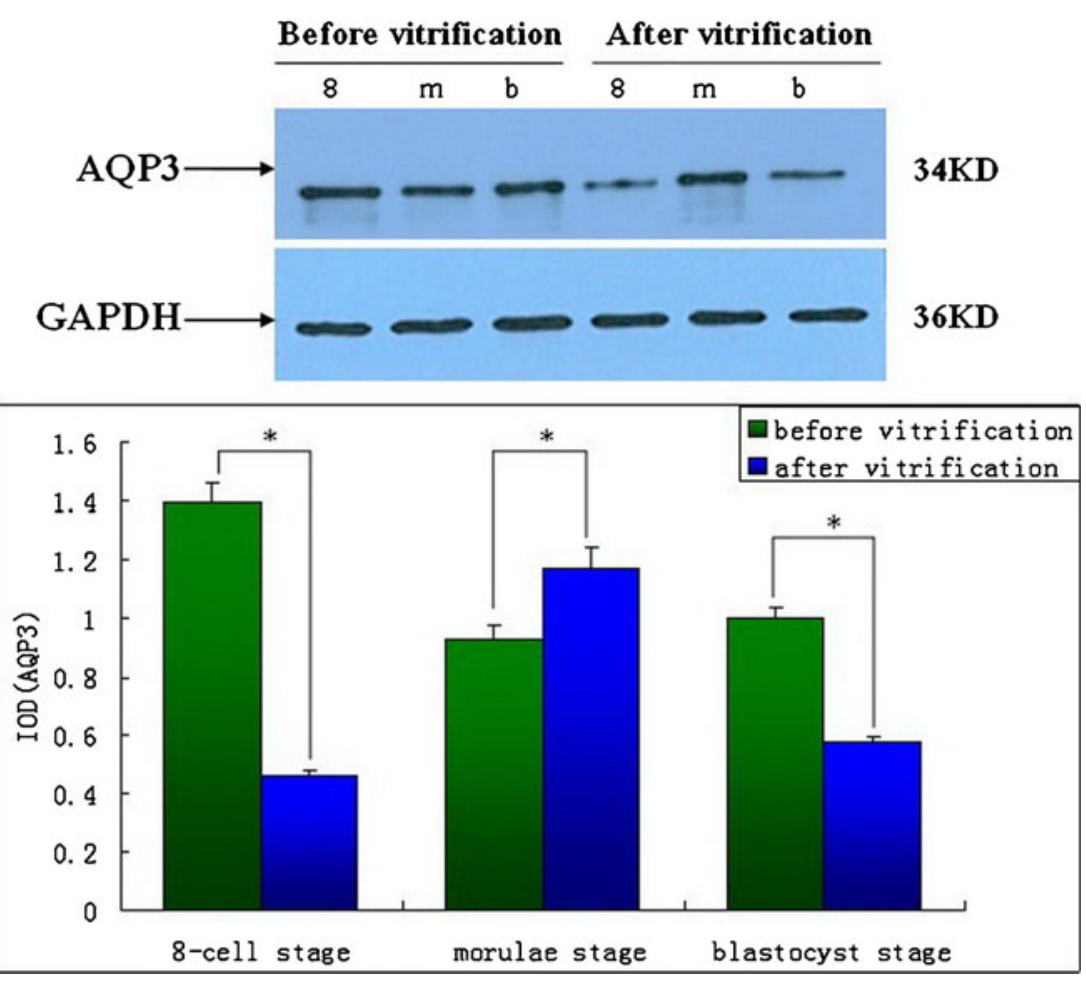

blastomere before and after vitrification. In early blastocysts, before and after vitrification, AQP3 was predominantly distributed over the plasma membrane and cytoplasm of trophoblastic cell, as well as blastocyst cavity. These results differ slightly from those reported by Barcroft (13), probably due to the different model species and detection methods. The observations reported in this study indicate that in $\mathrm{KM}$ mice 8-cell embryos, AQP3 is expressed not only in the cytoplasm but also in the karyotheca, then with stronger expression in the karyotheca relative to the cytoplasm.

Aquaporins are expressed in mouse 8-cell to blastocyst stage before and after vitrification. However, AQP3 was expressed at different sites at each embryonic stage before and after freeze-thawing, suggesting distinct functions at different developmental stages and different effects on vitrification. Before vitrification, AQP3 was mainly expressed in the karyotheca of 8-cell embryos, hinting that AQP3 functions in nuclear division during this period, and may play a role in fluid transport to organelles such as the mitochondria and golgiosome to maintain osmotic pressure balance. In contrast, AQP3 was predominantly expressed at the plasma membrane during the morula stage, suggesting that AQP3 is mainly involved in fluid exchange between embryos and the external environment and the formation of blastocyst cavity. In the early blastocyst stage, AQP3 was mainly distributed to the plasma membrane and cytoplasm of trophoblastic cell, as well as blastocyst cavity, suggesting that AQP3 may play a role in the differentiation of blastocyst and maintain osmotic pressure balance. Moreover, it may involve in blastocyst implantation, acting on same important processes such as blastocyst's position, adhesion, invasion, et al. These expression sites changed dramatically after freeze-thawing, a process associated with major shifts in osmotic pressure. After vitrification, AQP3 was mainly distributed to the cytoplasm and plasma membranes in all three stages of embryos examined, suggesting that AQP3 may mediates transmembrane fluid exhange during freeze-thawing to maintain stable osmotic pressure of embryos in hypertonic fluid.

Previous animal studies concluded that cellular freezing is a dehydrant procedure indeed, while thawing meaning the reabsorption of water into cell. The major damage to embryos induced by freeze-thawing was due to intracellular and extracellular ice formation. Compared to slow-freezing, embryonic vitrification effectively avoids such injuries, but the dramatic changes in osmotic pressure and the excessive high concentration of cryoprotectant required can still damage embryonic cells during vitrification [14-16], thus reducing embryonic reproductive potential. Now, except for AQPs, not any other substance which could mediate water transmembrane movement had been found yet [16]. A previous study [17] revealed that changes in the expression of AQP mRNAs and shifts in protein location were major stress responses to hypertonic solution treatment before embryonic implantation. Our results revealed that the distribution of AQP3 protein in mouse embryos can change rapidly during vitrification and that these specific patterns of expression and redistribution depend on developmental stage. We suggest that AQP3 contributes to embryo protection during freeze-thawing. 
Western blot analysis revealed that AQP3 expression was obviously detected in embryos of all stages before and after vitrification. The results indicated that the expression level of AQP3 in 8-cell embryos and blastocysts after vitrification was lower than before vitrification, while the expression of AQP3 in morulae was higher after vitrification, suggesting that AQP3 probably plays an important role in fluid exchange during this particular stage.

In the morula stage, blastomere becomes bound tightly together, with the narrowing of cellular gap and the formation of reticular structure of bounded cellular membrane. According to result of this study, after thawing, AQP3 was found mainly located on cell membrane of the once cryopreserved morula with higher expression than before. This indicates that in the presence of hypertonic cryoprotectants, AQP3 seems to be the main media of fluid exchange, stimulated to undergo protein localization and enhance expression. In this way, embryo is able to endure changes in osmotic pressure during cryopreservation and thawing since water and cryoprotectants can flow into and out of blastomeres effectively [18]. Besides, we speculate that other AQP subtypes may play a more dominant role in the transport and distribution of fluids during freeze-thawing of blastocyst stage and 8-cell stage.

To our knowledge, this is the first study compared the distribution and expression level of AQP3 in mouse 8-cell to blastocyst embryo stage before and after vitrification. We make the following conclusions. First, AQP3 expression varies depending on the development stage, indicating that AQP3s actively participate in early embryonic development. Second, AQP3 likely plays an active role in maintaining osmotic pressure balance during freeze-thawing of early embryos, especially morulae. Future studies will focus on the precise molecule mechnisms governing AQP3 expression and localization during freeze-thawing of morulae. In addition, we will investigate the expression and function of other AQPs during embryonic development to further elucidate the role of AQPs in protecting early stage embryos during freezethawing. These studies could lead to more effective methods for enhancing the viability of freeze-thawed embryos.

Acknowledgments The authors thank the Embryology Team of Reproductive Medicine Center at the third affiliated hospital of Guangzhou Medical University for its invaluable assistance in developing the vitrification protocol used in this study. They also thank the Animal Center of Guangzhou Medical University for providing SPF animal laboratory to carry out the animal experiments.

Open Access This article is distributed under the terms of the Creative Commons Attribution License which permits any use, distribution, and reproduction in any medium, provided the original author(s) and the source are credited.

\section{References}

1. Cho YS, Svelto M, Calamha G. Possible functional implications of aquapor in water channels in reproductive physiology and medically assisted procreation. Cell Mol Biol (Noisy-le-grand). 2003;49(4):515-9.

2. Maggio M, Lauretani F, Ceda GP, Bandinelli S, Basaria S, Paolisso $\mathrm{G}$, et al. Association of hormonal dysregulation with metabolic syndrome in older women: data from the InCHIANTI study. Am J Physiol Endocrinol Metab. 2008;292(1):E353-8.

3. Benga G. Water channel proteins (later called aquaporins) and relatives: past, present, and future. IUBMB Life. 2009;61:112-33.

4. Verkman AS. More than just water channels: unexpected cellular roles of aquaporins. J Cell Sci. 2005;118(15):3225-32.

5. Huang HF, He RH CC, Zhang Y, Meng QX, Ma YY. Function of aquaporins in female and male reproductive systems. Hum Reprod Update. 2006;12(6):785-95.

6. Seki S, Kouya T, Hara T, Valdez Jr DM, Jin B, Kasai M, et al. Exogenous expression of rat aquaporin-3 enhances permeability to water and cryoprotectants of immature oocytes in the zebrafish (Danio rerio). J Reprod Dev. 2007;53(3):597-604.

7. Edashige K, Ohta S, Tanaka M, Kuwano T, Valdez Jr DM, Hara T, et al. The role of aqnaporin 3 in the movement of water and cryoprotectants in mouse morulae. Biol Reprod. 2007;77(2):36575.

8. Edashige K, Sakamoto M, Kasai M. Expression of mRNAs of the aquaporin family in mouse oocytes and embryos. Cryobiology. 2000;40(2):171-5.

9. Richard C, Gao J, Brown N. Aquaporin water channcl genes are differentially expressed and regulated by ovarian steroids during the pri-implantation period in the mouse. Endocrinology. 2003;144(4):1533-41.

10. Offenberg H, Barcroft LC, Gavency A, Viuff D, Thomsen PD, Watson AJ. mRNAs encoding aquaporins are present during murine preimplantation development. Mol Reprod Dev. 2000;57(4):323-30.

11. Nagy VA, Gertsenstein M, Vintersten K. Manipulating the mouse embryo: a Laboratory Manua 1 [M]. CSHL Press. 2003;124126:146-60.

12. Edashige K, Tanaka M, Ichimaru N, Ota S, Yazawa K, Higashino $\mathrm{Y}$, et al. Channel-dependent permeation of water and glycerol in mouse morulae. Biol Reprod. 2006;74(4):625-32.

13. Barcroft LC, Offenberg H, Thomsen P, Watson AJ. Aquaporin proteins in murine trophectoderm mediate transepithelial water movements during cavitation. Dev Biol. 2003;256(2):342-54.

14. Kuwayama M, Fujikawa S, Nagi T. Ultrasture of IVM-IVF bovine early blastocysts vitrified after eqilibration in gly1,2-propanediol using 2-step and 16-step procedures. Cryobiology. 1994;31(5):415-22.

15. Damien M, Luciano AA, Peluso JJ. Propannedilo-induced alterations in membrane integrity, metabolism and developmental prtential of mouse zygotes. Hum Reprod. 1989;4(8):969-74.

16. Dobrinsky JR. Cellular approach to cryopreservation of embryos. Theriogenology. 1996;45(1):17-26.

17. Bell CE, Larivière NM, Watson PH, Watson AJ. Mitogen-activated protein kinase(MAPK) pathways mediate embryonic responses to culture medium osmolarity by regulating Aquaporin 3 and 9 expression and localization, as well as embryonic apoptosis. Hum Reprod. 2009;24(6):1373-86.

18. Tao J, Tamis R, Fink K. Cyropreservation of mouse embryos at morula/compact stage. J Assist Reprod Genet. 2001;18:235-43. 